\title{
Gastrointestinal perforation in COVID-19 patients - case series and review of the literature
}

\author{
Deepak Verma $^{\mathrm{a},}{ }^{*}$, Sarthak Sharma ${ }^{\mathrm{a}}$, Lalit Kishore Garg ${ }^{\mathrm{a}}$, Aishwarya Tinaikar ${ }^{\mathrm{a}}$
}

${ }^{a}$ Department of Surgery, Dr. Sampurnanand Medical College, Jodhpur 342001, Rajasthan, India.

\begin{abstract}
Severe acute respiratory syndrome coronavirus-2 (SARS-CoV 2) involving the respiratory tract started in 2019 in Wuhan, China but spread to almost all parts of the world to the extent that it was declared a pandemic by WHO. As more and more cases are detected, the involvement of systems other than respiratory was observed and nearly 5 to $50 \%$ of patients have abdominal symptoms like pain and diarrhea. During the course of treatment, few patients developed a rare complication of gastrointestinal perforation. Three cases (two with colonic perforation and one with gastric perforation) of gastrointestinal perforation in COVID-19 positive patient are reported in the article with a review of the literature regarding the various hypothesis of intestinal perforation in these patients.

Keywords: COVID-19; gastrointestinal perforation; severe acute respiratory syndrome coronavirus-2 (SARS-CoV 2); Tocilizumab
\end{abstract}

\section{INTRODUCTION}

Severe acute respiratory syndrome coronavirus-2 (SARS-CoV 2) caused coronavirus disease and it had affected 5 crore world's population with 14 lakh deaths. Since the virus primarily affected respiratory systems, the initial phase of symptomatology revolved around respiratory symptoms like dry cough, fever, etc. due to the involvement of the lungs ${ }^{[1]}$. But as time passed, varied symptoms were increasingly noted either in addition to respiratory symptoms or in isolation like loss of smell and taste, myalgia, pain abdomen, diarrhea ${ }^{[2,}$

${ }^{3]}$. Lately, gastrointestinal symptoms with acute abdomen were observed in a few patients, and a diagnosis of gastrointestinal perforation was made.

As more and more studies poured in regarding the nature of Severe acute respiratory syndrome coronavirus -2 (SARS-CoV 2), theories were postulated

\footnotetext{
* Corresponding author: Deepak Verma

Mailing address: Department of Surgery, Dr. Sampurnanand

Medical College, Jodhpur 342001, Rajasthan, India.

E-mail: drdeepakv3@gmail.com

Received: 11 December 2020 / Accepted: 24 December 2020
}

regarding the pathogenesis of perforation. Presence of Angiotension-converting Enzyme (ACE 2) receptors in the stomach, duodenum, and rectum, thromboembolic phenomenon leading to ischemia of gut, neuroinvasive propensity of virus causing dilatation of gut, increase intraluminal pressure due to constipation and finally the rare side effect of Tocilizumab used for COVID-19 cases treatment were considered as causes for gastrointestinal tract perforation.

We are presenting a case report of three cases presenting as perforation during the course of COVID-19 disease along with a review of the literature regarding causes of such perforation.

\section{CASE REPORT}

Case 1

A 60 years old female was admitted with symptoms of dry cough, fever, and myalgia and tested positive for COVID-19 RT-PCR. She was a known case of hypothyroidism (on 75 microgram thyroxine per day). She was treated with steroids and Rivaroxaban and sent for home isolation after 7 days. On the $4^{\text {th }}$ day of her discharge, she complained of pain abdomen and distension. Examination revealed distension with guard- 
ing and rigidity all over the abdomen. Besides other investigations, COVID-19 RT-PCR was repeated which was positive. D-Dimer was $953 \mathrm{ng} / \mathrm{ml}$, LDH- $735 \mathrm{U} / \mathrm{L}$, PT was 30.6 seconds with 2.56 INR. IL-6, Ferritin, and other investigations were within normal limits. CT thorax was suggestive of multifocal areas of consolidation in bilateral peripheral lung fields - typical of COVID-19 with a severity score of $17 / 25$ (Figure 1). X-Ray chest

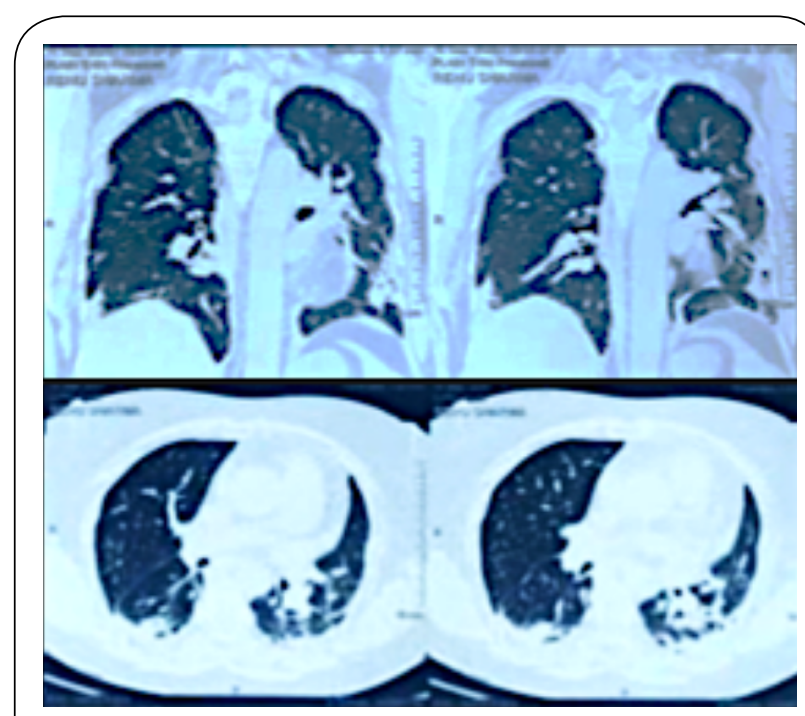

Figure 1: Chest CT scan of case 1 showing patchy consolidation of COVID-19 (Score 17/25).

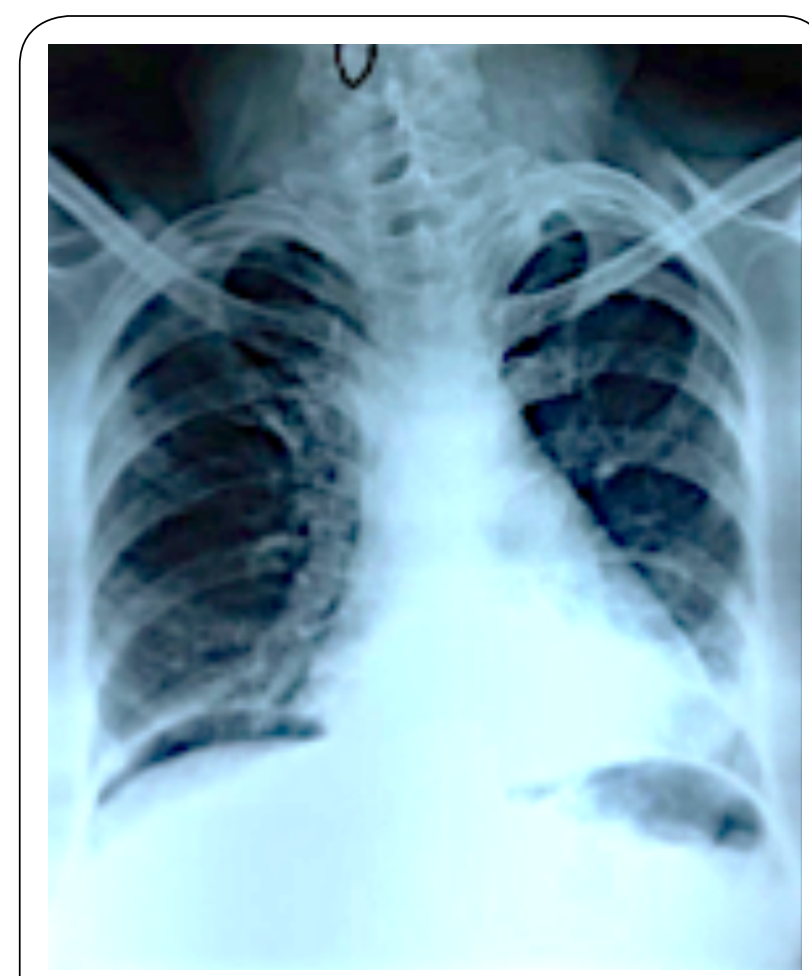

Figure 2: X-Ray Flat plate abdomen in erect posture showing gas under the diaphragm (Case 1). showed bilateral consolidation with gas under the diaphragm (Figure 2).

Exploratory laparotomy revealed a fecal-purulent collection of 2 liters with dense adhesion and $1 \mathrm{~cm}$ size perforation over the anterior wall of the upper onethird of the rectum. The surrounding rectal wall was oedematous. Repair of perforation with decompressing transverse loop colostomy was done. The patient was treated in ICU with antibiotics, fluconazole, low molecular weight heparin. Repeated RT-PCR for COVID-19 was negative on the $6^{\text {th }}$ post-operative day. She was discharged from the hospital on the $11^{\text {th }}$ post-operative day.

\section{Case 2}

A 24-year female was admitted to the Department of Obstetric with full-term pregnancy and labor pains. She was subjected to emergency Lower segment cesarean section. On the fifth postoperative day, she complained of severe pain abdomen, vomiting, and distension of the abdomen. The patient had tachycardia with abdominal distension and obliteration of liver dullness. Being immediate post-partum period, guarding and rigidity were not appreciated due to the lax abdominal wall. CT chest revealed atypical viral pneumonitis (Figure 3). X-Ray was showing a massive pneumoperitoneum (Figure 4) and COVID-19 RT-PCR was positive. D-Dimer was $3000 \mathrm{ng} / \mathrm{ml}$, IL6 - $127 \mathrm{pg} /$ $\mathrm{ml}$ and LDH was $800 \mathrm{U} / \mathrm{L}$. Patient was subjected to emergency exploratory laparotomy. There was $1.5 \mathrm{~L}$ of fecal-purulent fluid in the peritoneal cavity with adhesion and a $1.5 \mathrm{~cm}$ size perforation on the anterior wall of the caecum and edema of the caecal wall. Primary closure with decompressing loop ileostomy was done. Oxygenation supplementation, Meropenam antibiotics with low molecular weight heparin, and steroid were given. The patient recovered and was discharged with an ileostomy.

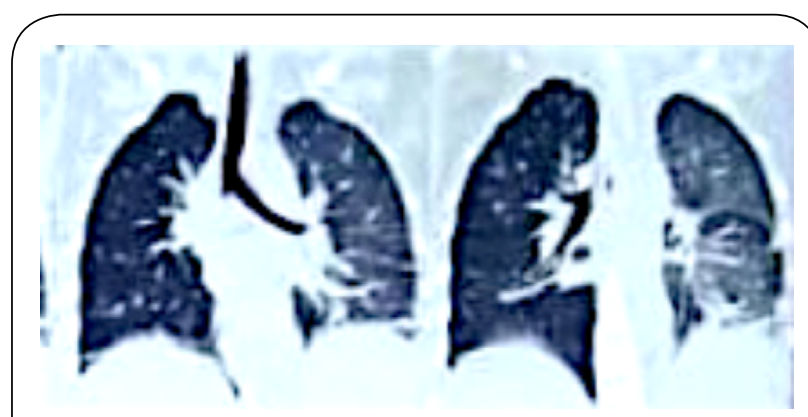

Figure 3: CT scan chest showing atypical viral pneumonitis (COVID-19) of case 2 . 


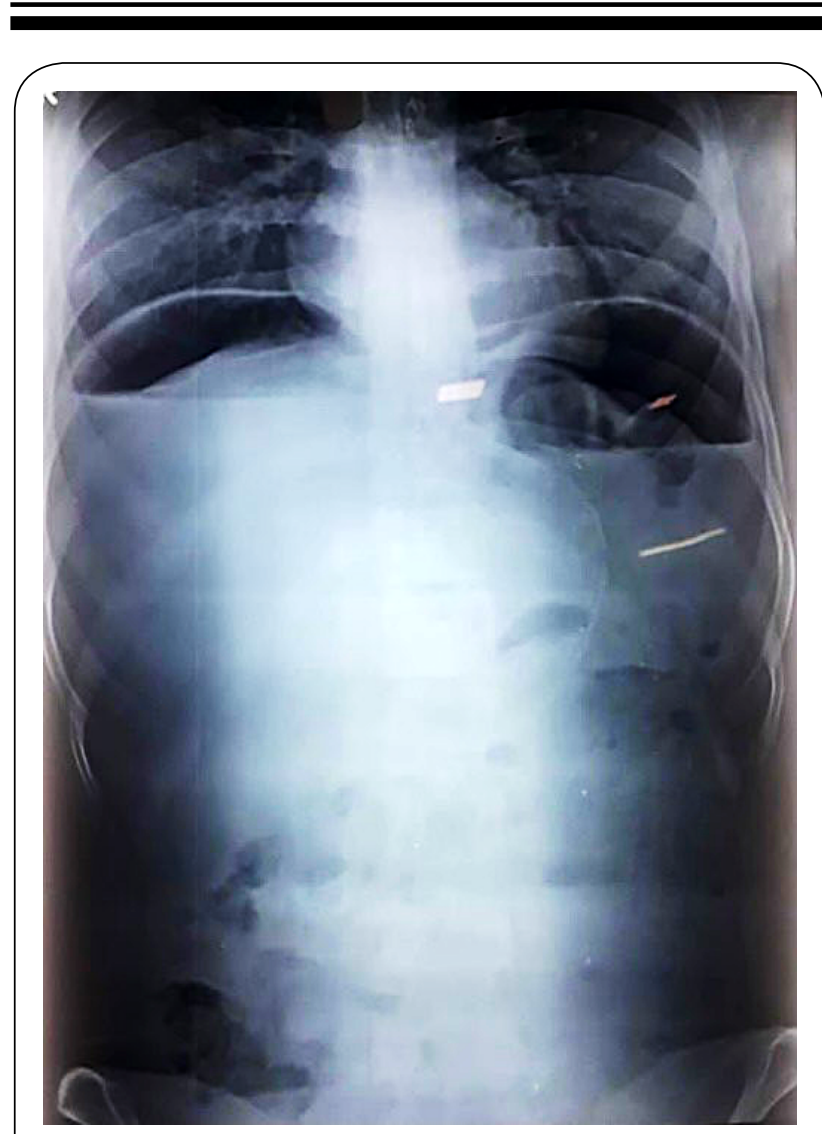

Figure 4: Presence of gas under diaphragm case 2.

\section{Case 3}

A 21-year-old male patient presented in a surgical emergency with a history of pain, fever, cough, vomiting, and distension of the abdomen. Clinical examination revealed a distended abdomen with guarding and rigidity along with pyrexia. No previous history of drug intake or acid peptic disease was present. X-Ray flat plate abdomen revealed gas under the diaphragm. COVID-19 RT-PCR was positive. Other routine investigations were normal except for an INR of 2.14. D-Dimer, IL-6, and LDH were marginally raised. Emergency exploratory laparotomy was done and there was inflamed thick omentum with $2 \mathrm{~L}$ of bile stained purulent fluid. $0.5 \mathrm{~cm}$ size perforation was seen on the anterior wall of the stomach at the pre-pyloric region. Closure of perforation with Graham's patch and LMWH, antibiotics, corticosteroids, and oxygen supplementation were administered. The patient was discharged on the $11^{\text {th }}$ post-operative day.

\section{DISCUSSION}

Severe acute respiratory syndrome coronavirus-2 (SARS-CoV 2) cause novel coronavirus disease. Although it started in Wuhan, China in 2019, now it had spread to all other parts of the world becoming a pandemic. India is the second most affected country across the world (nearly one billion cases) with a mortality of about $1.5 \%$. Since it primarily affects the respiratory system, symptoms such a fever, dry cough, dyspnea, and respiratory illness represent the most common manifestation ${ }^{[1]}$.

Since the virus and illness are relatively new, symptomatology is not very well understood and as the number of cases is increasing, various new symptoms like loss of smell and taste, etc. have also been reported. Approximately 5 to $50 \%$ of the cases have gastrointestinal symptoms ${ }^{[2]}$ like pain abdomen, diarrhea, and vomiting ${ }^{[3]}$. Detection of COVID-19 virus in stool ${ }^{[4]}$ and a potential fecal-oral transmission route has been suggested ${ }^{[5]}$. The various mechanism by which the gastrointestinal tract is affected in COVID-19 has been postulated. High expression of the angiotensin-converting enzyme (ACE) 2 receptor in the epithelium of the stomach, duodenum, and rectum ${ }^{[6]}$ with SARS-CoV 2 binding to these receptors to gain entrance to host cell ${ }^{[7]}$ is the key mechanism of involvement of gastrointestinal tract.

Studies have stated that critically ill patients with COVID-19 are more likely to have a hypercoagulable state, with the manifestation of intravascular coagulation due to local damage ${ }^{[8,9]}$. This may lead to ischemia of the entire gut with colonic perforation as a part of the septic and thromboembolic phenomenon caused by viral infection directly and indirectly.

SARS-CoV 2 RNA was mainly detected in the cytoplasm of gastrointestinal epithelial cells indicating infection and replication of the virus in these cells leading to local damage ${ }^{[10]}$.

Since the neuroinvasive propensity has been demonstrated as a common feature of coronavirus, a neuronal injury should be regarded as a possible pathogenetic mechanism ${ }^{[11]}$. This causes an imbalance of the autonomic innervation of the colon, altered colonic motility, and dilatation of the colon.

Critically ill ICU patients due to COVID-19 are mostly kept nil orally and can also have stress-related mucosal damage with ulceration. These patients become significantly constipated contributing to increased intraluminal pressure ${ }^{[12]}$. Colonic dilatation and increase intraluminal pressure can precipitate perforation of the gastrointestinal tract particularly the stomach, duodenum, and colon. Studies have demonstrated the detectable presence of coronavirus RNA in stools of infected patients ${ }^{[10,13]}$. Perforation in COVID-19 patients has been reported in various studies ${ }^{[2,14]}$. A combina- 
tion of factors like mucosal damage, ulceration, overdistension of the bowel, and increased intraluminal pressure contribute to the perforation of the bowel.

Tocilizumab, a monoclonal IL-6 receptor antibody, is used in rheumatoid arthritis and its use was identified as a risk factor associated with lower intestinal perforation, which had been reported as a rare but serious complication in a clinical trial of this drug ${ }^{[15]}$. The mechanism of intestinal perforation in patients receiving Tocilizumab is not fully understood, but prior diverticulitis has been noted as a risk factor ${ }^{[15]}$. Since there is no elevation of C-Reactive protein in these patients there is some delay in the diagnosis of perforation. Tocilizumab is also used in the treatment of cytokine release syndrome associated with severe COVID-19 ${ }^{[16]}$. Other authors have also warned about the possibility of the risk of intestinal perforation while using Tocilizumab in COVID-19 patients ${ }^{[17]}$. However, in the cases reported in this study, Tocilizumab was not used in any patient for COVID-19 treatment.

Diagnosis of gastrointestinal perforation can easily be made by history of pain, distension of abdomen, guarding and rigidity, and radiographic evidence of pneumoperitoneum. In a clinically stable patient, CT abdomen is more sensitive investigation along with CT chest to predict outcome post-surgery. Early operative intervention carries a better prognosis. Delay in the diagnosis of gastrointestinal perforation is associated with a poor prognosis due to compromised respiratory status, septicemia, edema of gut precluding optimum closure of gut, and high incidence of the thromboembolic phenomenon. However, in cases diagnosed later with the poor general condition, drainage of the peritoneal cavity at the bedside with non-operative conservative management can be attempted although with a guarded prognosis.

\section{DECLARATIONS}

\section{Authors' contributions}

Deepak Verma participated in surgical treatment and wrote the article. Sarthak Sharma carried out treatment and recordkeeping and data acquisition. Lalit Kishore Garg checked the article and supervised the patient's care. Aishwarya Tinaikar followed the patient in outcome point.

\section{Conflict of interest}

The authors declared that there are no conflicts of interest to disclose.

\section{Consent for publication}

Written consent was obtained from the individual case for publication of images used in this paper.

\section{REFERENCES}

1. Zhu, N., Zhang, D., Wang, W., Li, X., Yang, B., Song, J., ... \& Niu, P. (2020). A novel coronavirus from patients with pneumonia in China, 2019. New England Journal of Medicine.

2. Neto, I. J. F. C., Viana, K. F., da Silva, M. B. S., da Silva, L. M., de Oliveira, G., da Silva Cecchini, A. R., ... \& Robles, L. (2020). Perforated acute abdomen in a patient with COVID-19: an atypical manifestation of the disease. Journal of Coloproctology.

3. Kotfis, K., \& Skonieczna-Żydecka, K. (2020). COVID-19: gastrointestinal symptoms and potential sources of 2019-nCoV transmission. Anaesthesiol Intensive Ther 2020; 52 (1).

4. Jin, X., Lian, J. S., Hu, J. H., Gao, J., Zheng, L., Zhang, Y. M., ... \& Yu, G. D. (2020). Epidemiological, clinical and virological characteristics of 74 cases of coronavirus-infected disease 2019 (COVID-19) with gastrointestinal symptoms. Gut, 69(6), 1002-1009.

5. Gu, J., Han, B., \& Wang, J. (2020). COVID-19: gastrointestinal manifestations and potential fecal-oral transmission. Gastroenterology, 158(6), 1518-1519.

6. Zhao, D., Yao, F., Wang, L., Zheng, L., Gao, Y., Ye, J., ... \& Gao, R. (2020). A comparative study on the clinical features of COVID-19 pneumonia to other pneumonias. Clinical Infectious Diseases.

7. Wong, S. H., Lui, R. N., \& Sung, J. J. (2020). Covid-19 and the digestive system. Journal of gastroenterology and hepatology, 35(5), 744-748.

8. Song, Y., Liu, P., Shi, X. L., Chu, Y. L., Zhang, J., Xia, J., ... \& Wang, M. Y. (2020). SARS-CoV-2 induced diarrhoea as onset symptom in patient with COVID-19. Gut, 69(6), 1143-1144.

9. Wang, W., Gao, R., Zheng, Y., \& Jiang, L. (2020). COVID-19 with spontaneous pneumothorax, pneumomediastinum and subcutaneous emphysema. Journal of travel medicine, 27(5), taaa062.

10. Tian, Y., Rong, L., Nian, W., \& He, Y. (2020). gastrointestinal features in COVID-19 and the possibility of faecal transmission. Alimentary pharmacology \& therapeutics, 51(9), 843-851.

11. Conde, G., Pájaro, L. D. Q., Marzola, I. D. Q., Villegas, Y. R., \& Salazar, L. R. M. (2020). Neurotropism of SARS-CoV 2: Mechanisms and manifestations. Journal of the neurological sciences.

12. Kangas-Dick, A., Prien, C., Rojas, K., Pu, Q., Hamshow, M., Wan, E., ... \& Wiesel, O. (2020). Gastrointestinal perforation in a critically ill patient with COVID-19 pneumonia. 
SAGE open medical case reports, 8, 2050313X20940570.

13. Zhang, W., Du, R. H., Li, B., Zheng, X. S., Yang, X. L., Hu, B., ... \& Zhou, P. (2020). Molecular and serological investigation of 2019-nCoV infected patients: implication of multiple shedding routes. Emerging microbes \& infections, 9(1), 386-389.

14. De Nardi, P., Parolini, D. C., Ripa, M., Racca, S., \& Rosati, R. (2020). Bowel perforation in a Covid-19 patient: case report. International Journal of Colorectal Disease, 1.

15. Strangfeld, A., Richter, A., Siegmund, B., Herzer, P., Rockwitz, K., Demary, W., ... \& Listing, J. (2017). Risk for lower intestinal perforations in patients with rheumatoid arthritis treated with tocilizumab in comparison to treatment with other biologic or conventional synthetic DMARDs. Annals of the rheumatic diseases, 76(3), 504510.

16. Zhang, C., Wu, Z., Li, J. W., Zhao, H., \& Wang, G. Q. (2020). The cytokine release syndrome (CRS) of severe COVID-19 and Interleukin-6 receptor (IL-6R) antagonist Tocilizumab may be the key to reduce the mortality. International journal of antimicrobial agents, 105954.

17. Vikse, J., \& Henry, B. M. (2020). Tocilizumab in COVID-19: beware of risk of intestinal perforation. International Journal of Antimicrobial Agents. 